\title{
Basic Structure Content Scaling
}

\author{
Douglas N. Jackson \\ The University of Western Ontario
}

\author{
Edward Helmes \\ London Psychiatric Hospital
}

A basic structure approach is proposed for obtaining multidimensional scale values for attitude, achievement, or personality items from response data. Based on conventional components analysis, and described in terms of singular value or EckartYoung decomposition of a data matrix, basic structure scaling yields projections of items upon axes in the person space, equivalent to obtaining component scores for items, with component loadings associated with individuals. Unlike multidimensional scaling methods, the scaling of large sets of stimuli is practical and judgments of items are obviated. In attitude and personality item scaling. the technique permits the unconfounding of scale values due to response bias and to content. It also permits the partitioning of item indices of popularity or difficulty among a number of relevant dimensions, a property of possible relevance to tailored or adaptive testing.

Ever since Thurstone's (1929) classic work in attitude measurement, there has been a recurring need for item scale values, representing the locations of items on underlying content dimensions. Such information is useful for a number of practical and theoretical applications, for example, to construct attitude, personality, and ability measures so that different points along content dimensions are appraised; to identify the polarity of items or other stimuli

APPLIED PSYCHOLOGICAL MEASUREMENT

Vol. 3, No. 3 Summer 1979 pp. 313-325

(C) Copyright 1979 West Publishing Co. with respect to one or more dimensions; and as a basis for investigating processes underlying responses.

Torgerson (1958) distinguished between two broad approaches to the types of data from which the scale values of interest-judgments and responses-could be derived. For a judgment, "the subject responds to a stimulus with respect to its relation among other stimuli in the defined continuum"; whereas "in the response approach, the task set for the subject is to respond to a stimulus on the basis of the position of the stimulus in relation to the subject's own position with respect to the attribute" (p. 48). In a judgment, the position of the judge with regard to the stimulus is ignored over his/her perceptions of the relations among stimuli. In a response, both the judge's perception of stimuli and his/her (perceived) position among the stimuli are relevant. Therefore, differences in scale values may appear when data from the two types of data are compared.

For example, there is evidence (Boyd \& Jackson, 1966) that multivariate response methods applied to a set of attitude items yield a markedly different structure from that provided by multidimensional scaling of the judgments of similarity of the same items. The former present a less clear structure: one affected by response bias, a component not present in the judgments. Such a component may not only be of interest in 
itself, but may also be preferred so that its effect can be minimized in order to study more accurately the dimensions of content. Thus, it may be preferable to exercise a degree of control over the selection of dimensions in a solution based upon responses, which is unnecessary in a method using judgments (e.g., the techniques commonly referred to as multidimensional scaling).

Why then, would there be interest in scaling objects from responses rather than using judgments? First, a researcher may wish to determine if individuals respond to constructs in the same manner in which they judge them. Second, there are cases in which the collection of judgments is impractical. For example, to scale the 352 items on each of the 22 dimensions represented in Form E of the Personality Research Form (PRF-E; Jackson, 1974) would require 7,744 Likert-type judgments or 61,176 paired comparison judgments of similarity. In contrast, only 352 responses are required by the method described here. In addition, judgments may in turn affect the dimensionality of the relevant space. Torgerson (1965) reported that subjects' judgments of certain physical stimuli added dimensions of complexity not used when the stimuli were constructed.

Most methods of analyzing response data are based on factor or components analysis (Shepard, 1972), as is the procedure described here, which also employs a data matrix generated from responses to a set of items. It is intended for the scaling of the items of large multiscale personality and attitude questionnaires, although it is potentially useful for other types of assessment devices as well.

The work described here bears similarities to methods previously described. The logic of the method is described by Tucker (1955), Bennett (1956), and Coombs and Kao (1960) as applied to judgments of preferences. Slater (1960) describes a multidimensional model, which is also applied to preference judgments, very similar to the one dealt with here. Carroll (1972) does the same for the case of dominance judgments. A case applicable to the incidence of categories of attributes, analogous to responses, is described by Hill (1974). With the exception of the last paper, all previous discussions have been in terms of judgments, whereas the present method deals with responses, as defined earlier. It is recognized that there are formal similarities between scaling models applicable to judgments and responses (Nishisato, 1978). The difference between this approach and most of those mentioned above lies in the nature of the data. Responses and judgments from the same group of individuals may give rise to somewhat different multidimensional spaces. The present method concentrates on the space arising from responses.

The problem of representing different response patterns among respondents is approached here by considering each respondent as a vector in a multidimensional space (cf. Jackson \& Messick, 1963). Each respondent is assumed to respond to each item with respect to a one-dimensional attribute, namely, how true or characteristic the item is of the person. Respondents may differ among themselves in their pattern or ordering of true and false responses. Rather than seeking similarities among items or tests, the similarities are sought among respondents. Assume that a set of personality items was drawn from scales for aggression, dominance, and exhibition and that these items varied in desirability. Persons responding to these items might present different patterns of true and false responses, depending on their perception of these attributes in themselves and upon the weight accorded desirability in responding. Different response patterns permit the isolation of different types or clusters of individuals with regard to the personality traits assessed. A type or cluster is represented by a vector extending in some particular direction within the multidimensional array of item responses such that the vector best represents that type. Item projections on each such vector re- 
present scale values for each identified type. Thus item responses can be used to scale those same items.

A similar rationale underlies vector models of preference judgment or of factor analysis of ratings of traits with respect to a single multidimensional criterion, such as desirability (Messick \& Jackson, 1972). Unlike classical unidimensional scaling models, such a vector model does not require that the relevant dimension(s) be specified in advance. Unlike some multidimensional scaling models, judgments of interstimulus distances are not required by the basic structure approach. Yet, it is well known that judgment data can be scaled using a vector model, just as response data can be scaled using a point model. The advantages of the basic structure approach lie in its use of responses. Normally, this means less demand upon the subject's time and attention, since fewer responses are required than judgments, as outlined earlier. In addition, analysis of responses to items may provide different information from that provided by judgments.

Nevertheless, it would normally be expected that much the same dimensions would appear from both types of data. The appearance of the same dimensions from both sources of data-judgments and responses-has been considered a necessary condition for accepting the generalizability of the dimensions (Stewart, 1974).

The rationale for the scaling procedure is developed formally as follows.

\section{Definition of Notation}

In general, the notation follows that of Horst (1965).

$X$ is an item $(N)$ by person $(n)$ matrix.

$Z$ is $X$, column standardized, with mean of zero and unit variance, scaled by $1 / \sqrt{ } N$ so that $R=Z^{\prime} Z$. This is different from conventional factor analytic practice, in which items are standardized.
$P \quad$ and $Q$ are the left and right basic orthonormals, respectively, of $Z$.

$\Delta \quad$ is the basic diagonal of $Z$.

$T$ is a $k$ by $k$ orthonormal transformation matrix, where $T^{\prime} T=T T^{\prime}=I$, and where $k$ is the number of dimensions.

$Y$ is an $N$ by $k$ matrix of item scale values with respect to $k$ dimensions.

\section{Description of the Method}

The procedure is a form of conventional components analysis. It is most parsimoniously described in terms of a singular value or EckartYoung decomposition, but this is not necessary. Interest, in this case, is in the projections of items upon axes of the person space. In traditional usage, this is equivalent to obtaining component scores for items, with component loadings associated with individuals. This is the critical difference between this approach and conventional methods of analyzing response data (Shepard, 1972) and vector preference models.

Using a singular value decomposition routine (Businger \& Golub, 1969),

$$
Z=P \Delta Q^{\prime}
$$

Then, perform an orthogonal Procrustes transformation of $k$ columns of $P$ (e.g., Schönemann, 1966; Ten Berge, 1977).

$$
Y=P T
$$

where $Y$ is the best least-squares approximation to the hypothesis or target matrix. Generally, this matrix will consist of the scoring key for the test or the best estimation of the allocation of the items to a priori dimensions. Alternatively, an oblique rotation may be used. If desired, the scale values can be rescaled to unit variance following rotation.

If a singular value decomposition routine is not available, the same solution may be obtained by conventional procedures (Kaiser, 1962; Horst, 1965). 


$$
\mathrm{R}=\mathrm{Z}^{\prime} \mathrm{Z}
$$

Note that $R_{\mathrm{c}}$ in Equation 3 is not a correlation matrix.

$$
\begin{aligned}
& R=Q \Delta^{2} Q^{\prime} \\
& A=Q \Delta \\
& B=A T \\
& Y=Z B\left(B^{\prime} B\right)^{-1}
\end{aligned}
$$

Again, it should be noted that this $Y$ represents component scores for items which are interpreted as content scale values for items. $Y$ is not the matrix of component scores for individuals.

Alternatively, the rotation may be deferred and carried out directly upon the unrotated item content scores,

$$
P=Z A\left(A^{\prime} A\right)^{-1}
$$

which may be entered into Equation 2 to obtain item scale values.

\section{Illustration}

To illustrate the sc..1ng procedure, a set of 16 items was selected from 4 scales of the Jackson Personality Inventory (JPI; Jackson, 1976). The responses of 82 college students were used to determine the scale values.

The first step, after arranging the items into groups by scales, was to standardize the binary response matrix to remove respondent means and to yield unit variance for each respondent. In accordance with the equations, an item by respondent data matrix was assumed, and that standardization was by columns.

Next, the standardized data matrix was decomposed according to Equation 1 and the four largest singular vectors of the left-hand basic orthonormal matrix, $P$, were retained. Since four scales taken from the JPI were involved, four vectors were retained as the expected number of dimensions. In other circumstances, a different criterion for the number of dimensions might have been employed.

In this case, scale values were desired for the four dimensions corresponding to the scales. Therefore, the hypothesis matrix for the targeted rotation consisted of +1 for an item keyed true on a scale, -1 for an item keyed false on a scale, and 0 for an item not keyed on a scale. There was thus only one non-zero entry in any given row of the hypothesis matrix, and that non-zero entry denoted the scale on which the item was keyed. The best least-squares approximation to this hypothesis matrix was obtained through Schoneman's (1966) orthogonal Procrustes procedure. The resulting rotated matrix was then rescaled to unit variance by columns, giving the final scale values, as reported in Table 1.

Examination of the scale values reveals that in general, extreme scale values were associated with items on their keyed scale and that the scale values invariably reflected the direction of keying. Exceptions to this were congruent with item content. For example, Item 100 had a rather extreme scale value for Self-Esteem, but was keyed on Conformity. Item content, which deals with being uncomfortable if dressed differently in a social setting, was congruent with low SelfEsteem as defined by the JPI. Other conformity items, not highly related to social settings, did not have scale values as extreme on the selfesteem dimension.

One point of concern in the use of this method is the determination of the number of dimensions to be retained. One possibility would be the ase of the number of a priori scales in the inventory or, secondarily, the number of factors in the test, as determined by separate factor analytic studies. The use of the number of scales has conceptual advantages in that the relationship of an item to the scales of wellconstructed tests or questionnaires is well defined. If a test displays convergent and dis- 


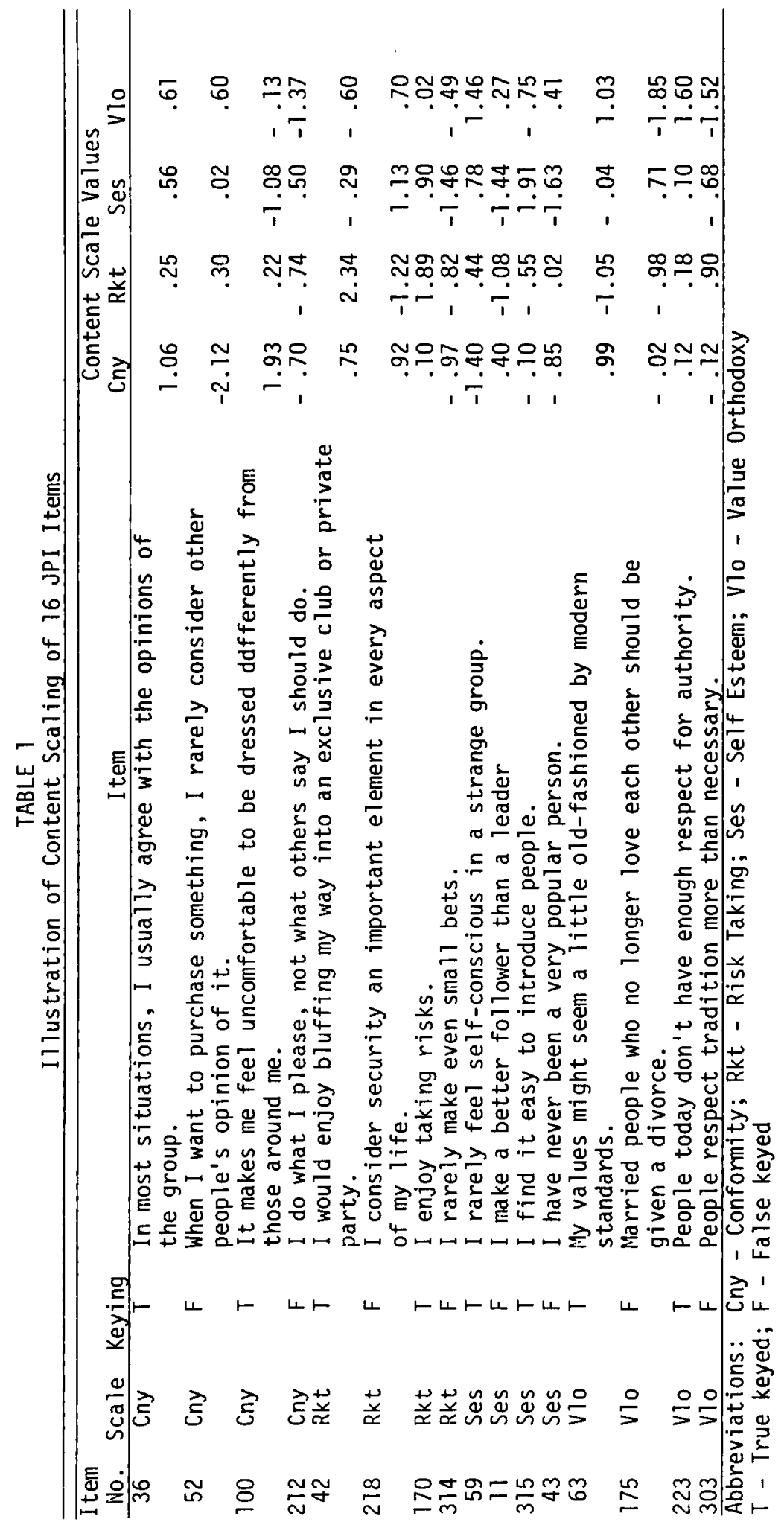


criminant validity and the suppression of irrelevant sources of variance, then each item should be related most strongly to its own scale.

For personality and attitude items, high content saturation on their keyed scale, together with a reduction in ambiguity of wording in items, is also a desirable item property in wellconstructed questionnaires. These two properties act to produce extreme scale values for items by yielding similar responses from persons possessing similar levels of the trait. Analogously, ability and achievement items having univocal properties in the sense that they assess skills or knowledge relevant to a single factor are considered desirable in multifactor test batteries. Ideally, such items will differentiate persons possessing particular levels of ability or achievement. As in the analogous case of Guttman scaling for unidimensional scales, the present approach is based on the expectation that persons having similar levels of a trait or ability will show similar response patterns to items associated with that trait. It would thus follow that the relative difficulty of items univocally associated with a particular factor would be associated with their scale values for dimensions corresponding to that factor.

It was suggested earlier that the hypothesis matrix might be based on the original item keying. But in exploratory work, such a key may not exist. The use of factors at either the scale or the item level provides an alternative source for the construction of an hypothesis matrix. This will normally involve the retention of substantially fewer components, which account for a smaller amount of total variance, but which may have higher utility for actual applications of the content scores. A conservative recommendation would be that the determination of the number of factors be based upon analysis of a different sample than that upon which content scores are to be derived.

Item factor analyses provide another source for the hypothesis matrix. A prime example is the Minnesota Multiphasic Personality Inventory (MMPI), from which several investi- gators have constructed scales not corresponding to the MMPI clinical scales. In cases such as these, the hypothesis matrix could be based upon the keying for the constructed scales or the hypotheses about item clusters, rather than the original scales.

The question now arises regarding the conditions under which this technique will yield useful scale values. Ideally, the use of this technique assumes that the scale meets certain minimum standards of internal consistency and of content homogeneity. Thus, the factorially complex MMPI clinical scales would not be appropriate. In addition, scales should meet the same criteria as those required of modern construct-oriented approaches to personality scale construction (Wiggins, 1973). Ideally (1) there should be a strong and demonstrable substantive and empirical relationship of an item to its own scale and to no other; (2) the scales should possess a degree of convergent and discriminant validity; (3) irrelevant sources of variance, whether due to irrelevant content or method variance, should be suppressed as much as possible; and (4) the items should have a moderate frequency of endorsement.

In addition, as a form of component analysis, this method of scaling requires the consideration of several specific points. The first is whether or not the basic structure method gives results different from an item factor analysis. The important differences between basic structure scaling and item factor analysis lie in the part of the basic triple product (Equation 1) used and in he standardization used. An item loading can be interpreted as representing the correlation between a set of responses and component scores. A content scale value defines the projection of the item upon the latent personality or ability dimension. These are logically distinct, in that they are geometrically separate, and also mathematically different in that they involve different combinations of the triple product resulting from the decomposition of the data matrix. In practice, component scores and loadings do not in general yield the same ordering of items on di- 
mensions. This is true even though the two sets of values may be based upon the same original data matrix, subject to different scalings. This does not deny that the two matrices are related in that, in the equations, they represent different scalings of the left-hand orthonormals.

The question of standardization and its effects upon the interpretation of factor analyses has been the subject of much confusion. It is generally accepted that the effects of standardization are to shift the origin and to expand or contract the axes of the multidimensional space (Ross, 1963). Therefore, a row standardization moves the origin of the space to the row centroid, while a column standardization moves it to the column centroid. In general, these two centroids are not identical and a different view of the multidimensional space is obtained in each case. In addition, the proportion of total variance removed by either a row or a column standardization differs, often by a substantial amount. This in turn can produce different interpretations (Gollub, 1968a, b; Tucker, 1968).

To illustrate that the direction of standardization does indeed produce different orderings of items, the set of oblique content scores for 22 PRF-E scales (as described later in this paper) were correlated with the corresponding set of oblique item component loadings reported by Helmes and Jackson (1977). These correlations ranged from .35 to .81 , with a median of .67 , indicating moderate correspondence. This is to be expected, as the two sets of values are based on a common 22-dimensional space. But the rank ordering of items within each dimension is far from identical in the two cases. A scaling of items based on an item factor analysis would therefore not agree very well with that given by basic structure content scores. The latter are based upon a definite rationale, including variance due to differences in item means in the derivation of the scale values, and are to be preferred over item factor loadings for purposes requiring the scaling of items.
A second point concerns any analysis of binary data, of which basic structure is only one example. There is an often-expressed belief that the decomposition of a correlation matrix based upon binary data leads to the presence of difficulty factors, that is, factors based solely upon differences in item frequency of endorsement. In contrast to this belief, Dingman (1958) empirically found difficulty factors to be of little or no practical consequence. Indeed, a search of the literature has revealed no bona fide example of this widely accepted phenomenon, aside from a very early report (Ferguson, 1941). McDonald and Ahlawat (1974) reported that such factors would be likely to appear only in cases in which items had both an extreme $p$-value and a nonlinear item trace line. It would thus appear that the importance of difficulty factors in the analysis of binary data has been overstated. A recent report by Hofmann and Gray (1978) is of further interest in this regard. They reported that component analysis was quite successful in recovering a perfect Guttman scale structure from binary data. In fact, it was marginally more successful than were factor analysis and image analysis.

Although originally intended for use with personality and attitude inventories, where the content domain is clearly multidimensional, this method is potentially useful in cognitive domains as well. For example, a problem in trigonometry might involve such ability factors as reading, vocabulary, spatial visualization, general reasoning, and number. Through the inclusion of appropriate subtests in a battery, the contribution of each of these components of ability to item difficulty could be assessed through examination of the appropriate content scale values. This in turn would imply that different item characteristic curves might be generated for a single item on each of the relevant ability dimensions.

This raises another point to be considered. Scaling of personality or attitude items most often deals with bipolar traits. Thus, the 
hypothesis matrix that is used to find the transformation matrix in Equation 2 is composed of +1 's, -1 's, and 0 's. This bipolarity of the traits provides a great deal of structure to the matrix and makes bipolar scale values meaningful. In contrast, dimensions of ability are usually conceptualized as unipolar. The correlation matrix of ability items almost invariably shows positive manifold. However, the basic structure scaling procedure forces bipolarity upon the items by placing the origin at the centroid of the person space. This makes the interpretation of scale values for ability items somewhat more difficult, as a conversion from bipolar to unipolar scale values must be made either implicitly or explicitly. The concept of partial $p$-values may be of use in dealing with this particular area of content.

\section{Partial $p$ values}

In a perfect Guttman scale, scale values are a function of item popularities (Green, 1956). But even with errorless data, if item responses are attributable to more than one dimension, unidimensional $p$ values may not be interpreted accurately as representing a single underlying content dimension.

A given a priori scale or test may contain a number of sets of items for which the item $p$ values in each set are attributable to different content or process dimensions or, alternatively, for which the item $p$ values are complex in that their endorsements or difficulties are each attributable to a number of dimensions in different proportions. Many investigators and test constructors implicitly make the convenient assumption of unidimensionality, but in certain instances, the attribution of $p$ value variance to distinct processes may be of some critical importance. For example, it is well known that the probability of endorsement of a personality item is a function of its desirability. Efforts to identify popularities attributable to their putative content would at least require separate identification of that portion due to desirability. In tailored or adaptive testing, item difficulties are employed as a basis for different item sequences. But if the test is designed to assess some unitary aptitude or achievement domain, it might be appropriate to employ item difficulty indices which are not due to the effects of extraneous sources of difficulty.

The scale values described in this paper can be interpeted as standardized item $p$ values. For a unidimensional, errorless test, the two would be highly correlated. For a multidimensional test, the standardized $p$ value could be reproduced by the sum of the item scale values on retained dimensions plus the residual components. Of course, item popularities or difficulties are not normally standardized, since it is more convenient and relevant to retain the metric of the unstandardized proportion of endorsements. If item $p$ values are required for separate dimensions, all of the formulas for item scale values apply, except that the unstandardized entity by attribute matrix, $X$, should be substituted for $Z$ in Equation 1. In this case, the elements of $Y$ in Equations 2 and 7 would represent "partial $p$ values." The sum of the elements in a given row of $Y$ plus the residual components would be equal to the proportion of the $n$ individuals endorsing that item or attribute. These elements would represent the popularities or difficulties of different items apportioned among the processes or content represented by the separate dimensions.

\section{Large Data Matrices}

The method is intended for large multiscale inventories in cases in which judgment methods require an excessive number of judgments. However, with a very large number of respondents, a data matrix can result which is too large for central storage in even a relatively large computer. In this case, the respondent by respondent correlation matrix will almost certainly exceed computer capacity, assuming more respondents than items. Alternative procedures do exist. For example, the decomposition may take place on the 
entity by entity covariance matrix and the equivalent results obtained by well-known transformations (Horst, 1965, p. 326; Messick \& Jackson, 1972; Tucker \& Messick, 1963). But even this may be too large to be practical for, say, large multiscale personality inventories (e.g., the example below of the 352 items of Form $\mathrm{E}$ of the Personality Research Form, PRF. $\mathrm{E}$, as administered to 1000 respondents). An alternative is to extend the item matrix into a components space defined by scales and persons.

Based upon scale keys or a priori clustering of item content, construct a data matrix $X_{s}$ of $L$ subscales and $n$ persons. There should be at least three items in each subscale and three subscales per scale. For example, each of the 22 scales of the PRF-E could be divided into four subscales of four items each. This would yield an $88 \times n$ data matrix.
Standardize this matrix, $X_{s}$, by columns, yielding $Z_{s}$. Note again that this removes the person mean, not the item mean. Proceed as described above to find a set of content scores for the subscales:

$Y_{S}=P_{S} T_{S}$

In addition, form the correlation matrix of items and subscales, $\boldsymbol{R}_{y s}$,

$$
R_{y s}=1 / n Z_{y} Z_{s}{ }^{\prime}
$$

where $Z_{y}$ is standardized the same as $Z$ in the previous section, but without the scaling factor.

In a manner analogous to Dwyer extension procedures (Dwyer, 1937; Tucker, 1971), proceed to obtain extension scale values for items on the scale constructs. Note, however, that this

TABLE 2

Scale Values and Items for the Achievement Scale

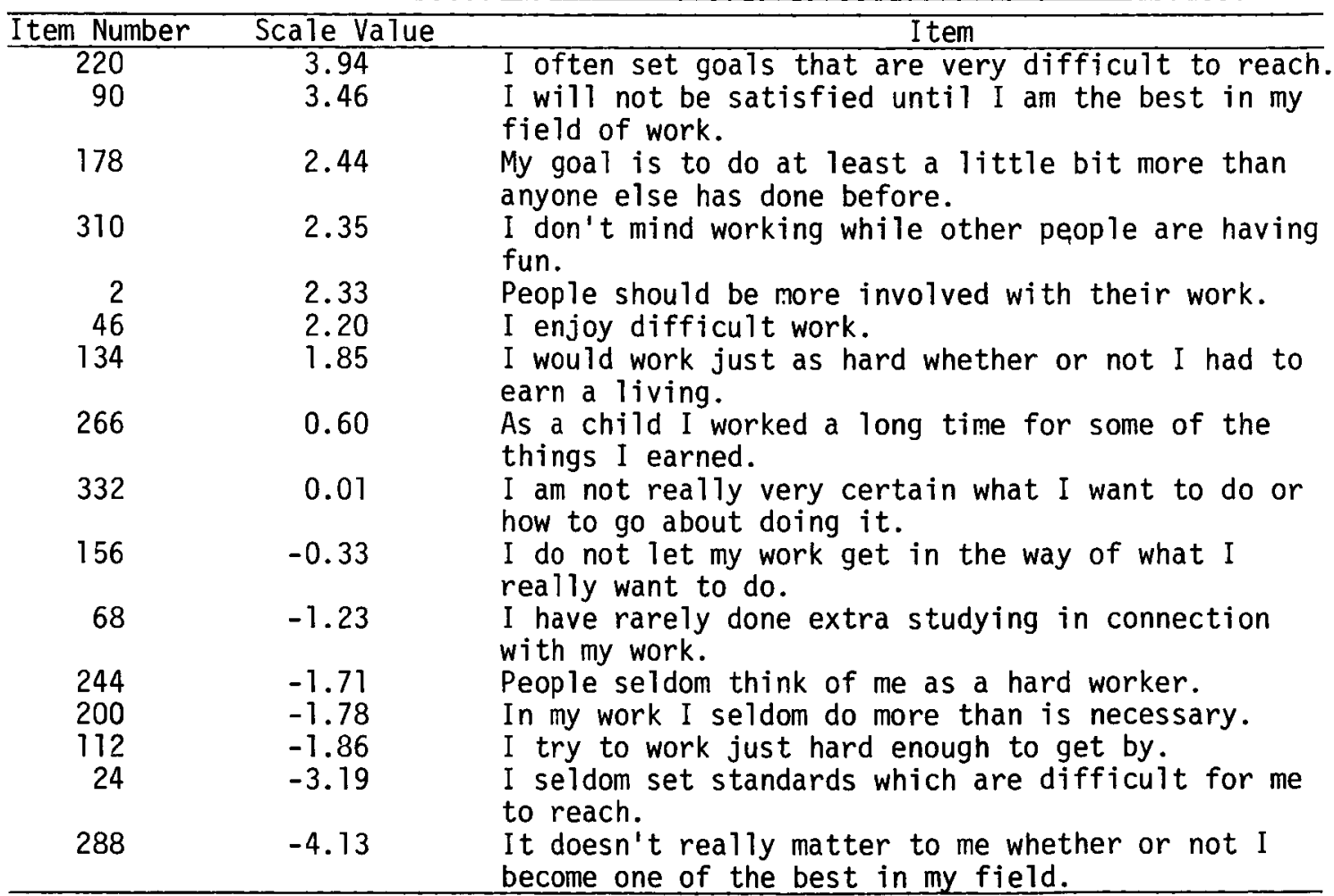


TABLE 3

Scale Values and Items for the Dominance Scale

\begin{tabular}{|c|c|c|}
\hline Item Number & Scale Value & I tem \\
\hline 317 & 4.73 & $\begin{array}{l}\text { I would like to be an executive with power } \\
\text { over others. }\end{array}$ \\
\hline 229 & 4.41 & $\begin{array}{l}\text { The ability to be a leader is very important } \\
\text { to me. }\end{array}$ \\
\hline 9 & 3.68 & $\begin{array}{l}\text { I feel confident when directing the activities } \\
\text { of others. }\end{array}$ \\
\hline 97 & 3.68 & $\begin{array}{l}\text { I try to control others rather than permit them } \\
\text { to control me. }\end{array}$ \\
\hline $\begin{array}{r}53 \\
141 \\
185\end{array}$ & $\begin{array}{l}3.47 \\
3.35 \\
2.34\end{array}$ & $\begin{array}{l}\text { I would like to be a judge. } \\
\text { I would like to play a part in making laws. } \\
\text { In an argument, I can usually win others over }\end{array}$ \\
\hline 273 & 2.06 & $\begin{array}{l}\text { I am quite effective in getting others to agree } \\
\text { with me. }\end{array}$ \\
\hline $\begin{array}{l}295 \\
251\end{array}$ & $\begin{array}{l}-2.24 \\
-2.44\end{array}$ & $\begin{array}{l}\text { I am not very insistent in an argument. } \\
\text { Most community leaders do a better job than I } \\
\text { could possibly do. }\end{array}$ \\
\hline $\begin{array}{r}339 \\
31 \\
119\end{array}$ & $\begin{array}{l}-2.56 \\
-3.48 \\
-4.03\end{array}$ & $\begin{array}{l}\text { I would not want to have a job enforcing the law. } \\
\text { I would make a poor military leader. } \\
\text { I don't like to have the responsibility for } \\
\text { directing the work of others. }\end{array}$ \\
\hline $\begin{array}{r}75 \\
207\end{array}$ & $\begin{array}{l}-4.23 \\
-4.38\end{array}$ & $\begin{array}{l}\text { I avoid positions of power over other people. } \\
\text { I feel uneasy when I have to tell people what } \\
\text { to do. }\end{array}$ \\
\hline 163 & -4.89 & I have little interest in leading people. \\
\hline
\end{tabular}

procedure involves the use of the left basic orthonormals rather than the more common right basic orthonormals,

$Y_{E}=R_{y s} Y_{s}\left(Y_{S} ' Y_{s}\right)^{-1}$

Thus item scale values may be obtained on a large item $\times$ subject matrix without the direct decomposition of this matrix. With relatively homogeneous scales, these values of $Y_{E}$ will be similar, but not in general identical, to those derived from Equation 2. There are two reasons for this: The first is due to the possible differences in the manner in which item and scale component analyses identify components; only if items on a scale form a perfect scale will the two be identical. The second lies in the differences in rotation. That is, the transformation matrices $T$ and $T_{s}$ do not rotate axes to precisely the same orientation.

\section{Example of Analysis of a Large Data Matrix}

Form $E$ of the PRF (Jackson, 1974) is composed of 352 items comprising 22 scales. The sample used to illustrate the scaling of this large inventory consisted of 214 college students drawn from U.S. colleges, as described in Helmes and Jackson (1977). These data were first scaled directly by means of Equation 2 . The hypothesis matrix consisted of the true-false scoring key with +1 for true-keyed items, -1 for false-keyed items, and 0 for items not keyed on a scale.

Complete results are not presented here for reasons of space, ${ }^{1}$ but Tables 2 and 3 give the

'Complete tables of orthogonal and oblique content scores for PRF-E have been deposited with NAPS. See NAPS Document No. 03198 for 53 pages of supplementary material. Order from ASIS/NAPS, c/o Microfiche Publica- 
TABLE 4

Mean Scale Values of Keyed and Non-Keyed Items

\begin{tabular}{|c|c|c|}
\hline Scale & 16 Keyed Items & 336 Non-Keyed Items \\
\hline$\overline{\text { Abasement }}$ & 1.88 & .71 \\
\hline Achievement & 2.08 & .69 \\
\hline Affiliation & 2.25 & .69 \\
\hline Aggression & 2.05 & .68 \\
\hline Autonomy & 2.30 & .66 \\
\hline Change & 2.43 & .63 \\
\hline Cognitive Structure & 2.28 & .68 \\
\hline Defendence & 2.40 & .71 \\
\hline Dominance & 3.51 & .51 \\
\hline Endurance & 2.24 & .67 \\
\hline Exhibition & 2.26 & .62 \\
\hline Harmavoidance & 3.32 & .55 \\
\hline Impulsivity & 2.49 & .65 \\
\hline Nurturance & 2.48 & .65 \\
\hline Order & 3.64 & .47 \\
\hline Play & 2.42 & .67 \\
\hline Sentience & 2.67 & .65 \\
\hline Social Recognition & 2.66 & .63 \\
\hline Succorance & 2.67 & .64 \\
\hline Understanding & 2.80 & .59 \\
\hline Infrequency & 2.47 & .69 \\
\hline Desirability & 1.75 & .69 \\
\hline
\end{tabular}

Note: False keyed items have been reflected.

scale values for items of the Achievement and Dominance scales. The values in Tables 2 and 3 have been taken from a matrix of content scores which have been rescaled to unit variance. As can be seen, the scale values are quite in accordance with item content and with direction of keying, as expected. PRF-E items were written to reflect high content saturation for the scale on which they are keyed (Jackson, 1974). Therefore, the scale values for items keyed on a scale should be substantially more extreme than for items not

(continued from previous page)

tions, P.O. Box 3513, Grand Central Station, New York, NY 10017. Remit in advance for each NAPS accession number. Institutions and organizations may use purchase orders when ordering. However. there is a billing charge of $\$ 5.00$ for this service. Make checks payable to Microfiche Publications. Photocopies are \$12.25. Microfiche are \$3.00. Outside the United States and Canada, postage is $\$ 3.00$ for photocopy or $\$ 1.00$ for fiche. keyed on that scale. In fact as seen in Table 4, this is the case. The procedure normally assigns negative scale values to false-keyed items. These have been reflected in Table 4 .

Item scale values were also obtained using the extension procedure in Equation 11 on the same set of subjects in order to evaluate the degree of similarity of the two sets of content scores. Four subscales were constructed for each PRF-E scale, each containing either four true-keyed items or four false-keyed items. This led to the decomposition of the $88 \times 88$ cross-products matrix, rather than a $352 \times 214$ data matrix. The reduction in the amount of computer central storage required is appreciable. The hypothesis matrix (consisting of $+1,-1$, and 0 as before) was then $88 \times 22$ rather than $352 \times 22$, as was the case when scaling items directly.

Simple linear correlations were calculated between the two sets of content scores for items 
keyed on a scale. Due to the high content saturation of these items, they would be most salient in the scaling procedure and have the most stable content scores. These correlations ranged from .73 to .99 , with a median of .96 .

This method of scaling provides a flexible means of scaling very large sets of stimuli such as are typically found in modern multiscale personality inventories. Even with large modern computers, it very often may be the only method of obtaining multidimensional scale values, given the difficulties of obtaining valid judgments from subjects with a large number of stimuli.

\section{References}

Bennett, J. F. Determination of the number of independent parameters of a score matrix from the examination of rank orders. Psychometrika, 1956, $21,383-393$.

Boyd, J. E., \& Jackson, D. N. An empirical evaluation of judgment and response methods in multivariate attitude scaling. American Psychologist. 1966, 21. 718. (Abstract)

Businger, P. A., \& Golub, G. H. Algorithm 358. Singular value decomposition of a complex matrix. Communications of the Association for Computing Machinery, 1969, 12, 564-565.

Carroll, J. D. Individual differences and multidimensional scaling. In R. N. Shepard, A. K. Romney, \& S. B. Nerlove (Eds.), Multidimensional scaling: Theory and applications in the behavioral sciences (Vol. 1). New York: Seminar Press, 1972.

Coombs, C. H., \& Kao, R. C. On a connection between factor analysis and multidimensional unfolding. Psychometrika, 1960, 25, 219-231.

Dingman, H. F. The relation between coefficients of correlation and difficulty factors. British Journal of Statistical Psychology, 1958, 11, 13-17.

Dwyer, P. S. The determination of the factor loadings of a given test from the known factor loadings of other tests. Psychometrika, 1937, 2, 173-178.

Ferguson, G. A. The factorial interpretation of test difficulty. Psychometrika, 1941, 6, 13-17.

Gollob, H. F. Confounding of sources of variation in factor-analytic techniques. Psychological Bulletin. 1968, 70, 330-344. (a)

Gollob, H. F. Rejoinder to Tucker's "Comments on confounding of sources of variation in factor- analytic techniques." Psychological Bulletin. $1968,70,355-360$. (b)

Green, B. F. A method of scalogram analysis using summary statistics. Psychometrika, 1956, 21 , 79-88.

Helmes, E., \& Jackson, D. N. The item factor structure of the Personality Research Form. Applied Psychological Measurement, 1977, 1, 185-194.

Hill, M. O. Correspondence analysis: A neglected multivariate method. Journal of the Royal Statistical Society, Series C: Applied Statistics, 1974, 23, 340-354.

Hofmann, R. J., \& Gray, W. M. On partialing a simplex out of binary data. Multivariate Behavioral Research, 1978, 13, 223-227.

Horst, P. Factor analysis of data matrices. New York: Holt, Rinehart, \& Winston, 1965.

Jackson, D. N. Personality Research Form Manual (Rev. ed.). Port Huron, MI: Research Psychologists Press, 1974.

Jackson, D. N. Jackson Personality Inventory Manual. Port Huron, MI: Research Psychologists Press, 1976.

Jackson, D. N., \& Messick, S. Individual differences in social perception. British Journal of Social and Clinical Psychology, 1963, 2, 1-10.

Kaiser, H. F. Formulas for component scores. Psychometrika, 1962, 27, 83-87.

McDonald, R. P., \& Ahlawat, K. S. Difficulty factors in binary data. British Journal of Mathematical and Statistical Psychology, 1974, 27, 82-99.

Messick, S., \& Jackson, D. N. Judgmental dimensions of psychopathology. Journal of Consulting and Clinical Psychology. 1972, 38. 418-427.

Nishisato. S. Optimal scaling of paired comparison and rank order data: An alternative to Guttman's formulation. Psychometrika. 1978, 43, 263-271.

Ross, J. The relation between test and person factors. Psychological Review, 1963, 70, 432-443.

Schönemann, P. H. A generalized solution of the orthogonal Procrustes problem. Psychometrika, 1966, 31, 1-10.

Shepard, R. N. A taxonomy of some principal types of data and of multidimensional methods for their analysis. In R. N. Shepard, A. K. Romney, \& S. B. Nerlove (Eds.), Multidimensional scaling: Theory and applications in the behavioral sciences (Vol. 1). New York: Seminar Press, 1972.

Slater, P. The analysis of personal preferences. British Journal of Statistical Psychology. 1960, 13. 119-135.

Stewart, T. R. Generality of multidimensional representations. Multivariate Behavioral Research. $1974,9,507-519$. 
Ten Berge, J. M. F. Orthogonal Procrustes rotation for two or more matrices. Psychometrika. 1977, 42, 267-276.

Thurstone, L. L. Theory of attitude measurement. Psychological Review, 1929, 36, 222-241.

Torgerson, W. S. Theory and methods of scaling. New York: Wiley, 1958.

Torgerson, W. S. Multidimensional scaling of similarity. Psychometrika, 1965, 30. 379-393.

Tucker, L. R. Description of paired comparison preference judgments by a multidimensional vector model (Research Memorandum 55-57). Princeton, NJ: Educational Testing Service, 1955.

Tucker, L. R. Comments on "Confounding of sources of variation in factor-analytic techniques." Psychological Bulletin. 1968, 70, 345-354.

Tucker, L. R. Relation of factor score estimates to their use. Psychometrika, 1971, 36, 427-436.

Tucker, L. R., \& Messick, S. An individual differences model for multidimensional scaling. Psychometrika. 1963, 28, 333-367.

Wiggins, J. S. Personality and prediction: Principles of personality assessment. Reading, MA: Addison-Wesley, 1973.

\section{Acknowledgments}

This work was supported by Canada Council Research Grant No. S75-0495-X2. We thank D. Chan for his comments on an earlier draft and I. Spence and $R$. A. Harshman for their suggestion to explore the effects of standardization. We also thank $P$. $M$. Bentler for his initial mention of partial $p$ values in this context and for supplying the data used in the first illustration. An earlier version of this paper was presented to the Society for Multivariate Experimental Psychology. University Park, PA, November, 1976.

\section{Author's Address}

Send requests for reprints or further information to Douglas N. Jackson, Department of Psychology, The University of Western Ontario, London, Ontario, Canada, N6A 5C2. 